\title{
Blastic plasmacytoid dendritic cell neoplasm with skin, bone marrow and CNS involvement: a case report
}

\author{
Kameta Imaeva, Ine Moors, Philip Vlummens, Fritz Offner (1); Jo Van Dorpe, Sofie \\ Verbeke (2); Jan Philippé, Barbara Denys (3) \\ 1, Department op Hematology, Ghent University Hospital, Belgium \\ 2, Department op Pathology, Ghent University Hospital, Belgium \\ 3, Department of Laboratory Medicine, Ghent University Hospital, Belgium
}
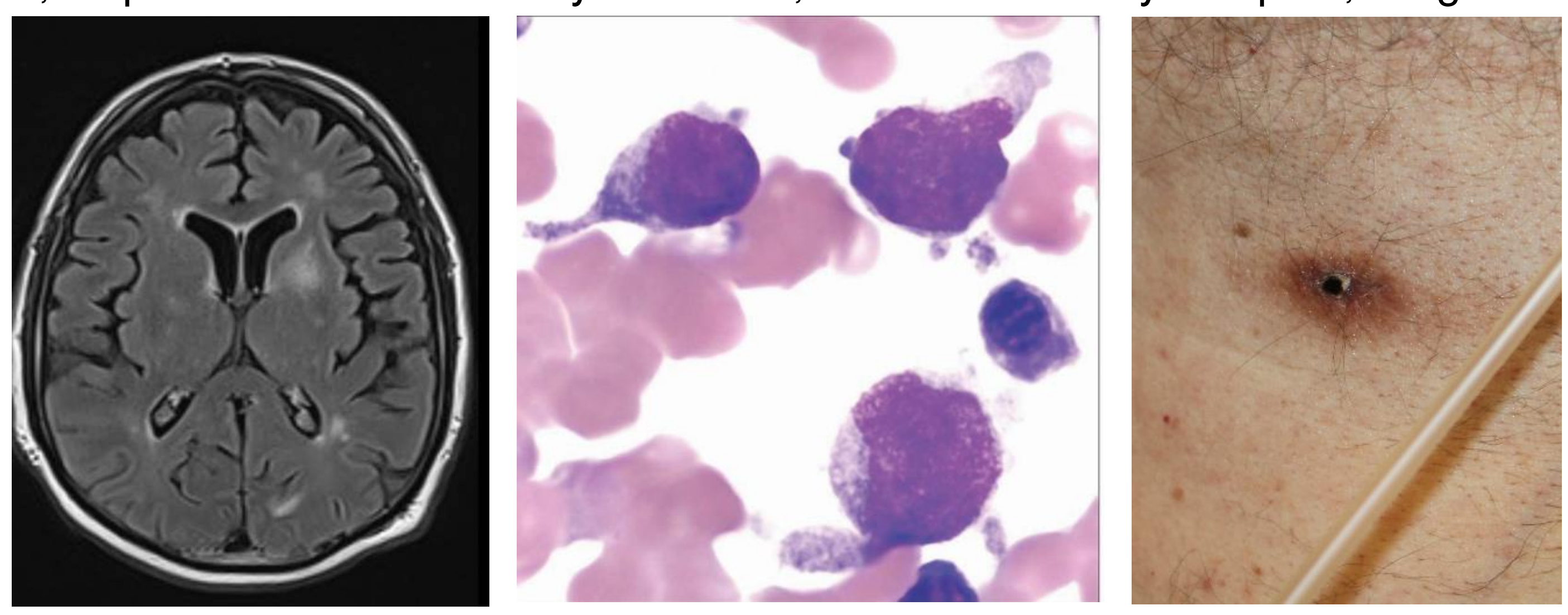

Case presentation

A 65-year-old man presented with constitutional symptoms and erythematous skin lesions. Blood analysis revealed pancytopenia, 2\% circulating blasts, DIC, liver function abnormalities and elevated LDH. Skin biopsy revealed a dermal infiltration with a diffuse monomorphous infiltrate of medium-sized blast cells coexpressing CD4+/CD56+/CD123+. Bone marrow aspirate showed myeloid and erythroid dysplasia, with $10 \%$ blasts, and a specific immunophenotype, CD4w+/CD15+/CD56+/CD34+/CD117++/CD123+/CD7++/CD38+/H LA-DR+/CD45 $\mathrm{w}+$ suggesting underlying MDS-EB2. Bone marrow biopsy showed a markedly hypercellular marrow (90\%) with CD4+/CD56+ small blasts negative for myeloid, NK- and lymphoid markers, and fibrosis grade 1. Cytogenetics revealed an aberrant karyotype with del(20q). Molecular analysis (NGS) of bone marrow revealed mutations in SRSF2 and ASXL1. Blastic plasmacytoid dendritic cell neoplasm (BPDCN) with cutaneous and bone marrow involvement was diagnosed. First AML induction therapy (cytarabine and idarubicine, $7+3$ ) induced a complete morphological and flow cytometric remission on bone marrow examination as well as negative skin biopsy. Screening lumbar puncture was normal. Shortly after starting the second AML induction therapy (high dose cytarabine and idarubicine), he developed rapidly progressive motor and sensory symptoms of the lower limbs with sphincter impairment. Radiological findings were compatible with acute transverse myelitis over Th2 to Th10. Broad-spectrum antimicrobial therapy was given but extensive search for microbiological or leukemic aetiology remained negative. Neurological symptoms temporarily resolved during aplasia and reappeared after hematologic recovery. Finally, a repeat lumbar puncture revealed CNS relapse with cells expressing the initial flowcytometric phenotype. At that time the patient was rapidly deteriorating and palliative treatment was started.
Discussion

BPDCN is a rare disease, which should be recognized by its characteristic immunophenotype (triple positive CD4+/CD56+/CD123 phenotype without other lineage-specific markers). Because of its rarity, there are no prospective trials with respect to treatment. AMLas well as ALL-like therapies have been used, and allogeneic or autologous HSCT appear to provide some survival benefit (1-3). Occult CNS disease should be actively screened for at diagnosis, and intrathecal prophylaxis and treatment can improve outcomes (4). It seems wise to combine "lymphoid" drugs like methotrexate and Lasparaginase which are able to prevent CNS involvement with "myeloid" drugs such as idarubicine (2). A combination of Lasparaginase, methotrexate and dexamethasone seems to prolong survival and induce remissions (7). New therapeutic approaches are being tested such as bortezomib (5) and SL-401 (anti-CD123 interleukin-3 receptor inhibitor) (6).

\section{Conclusion}

BPDCN is a very rare and clinically aggressive disease with a median survival of 10-19 months. Most cases (80-90\%) show an initial response to multiagent chemotherapy but relapses with resistance to drugs are regular. It has distinct immunophenotypic features (CD4+/CD56+/CD123+), with high frequency of cutaneous and bone marrow involvement, leukaemic dissemination and CNS involvement. CNS could be a persistent blast-cell sanctuary due to the limited penetration of cytostatic drugs across the blood-brain barrier in to the CSF and brain parenchyma (4). About $10-20 \%$ of cases of BPDCN are associated with or develop into other myeloid neoplasms such as CMML, MDS and AML. It is separately listed in the group of acute myeloid leukemias and related precursor neoplasms since WHO 2008.

1. Pagano et al. Blastic plasmacytoid dendritic cell neoplasm with leukemic presentation: an Italian multicenter study. Haematologica 2013; 98 (2).

2. Poret et al. How to treat blastic plasmacytoid dendritic cell neoplasm (BPDCN) patients: results on 86 patients of the French BPDCN network. ASH abstracts 2015 .

3. Tomohiro et al. Long-term survival following autologous and allogeneic stem cell transplantation for blastic plasmacytoid dendritic cell neoplasm. Blood 2015; 125 (23).

4. Martin-Martin et al. High frequency of occult central nervous system involvement in blastic plasmacytoid dendritic celle neoplasia at diagnosis: role for intrathecal prophylaxis? EHA abstracts 2016.

5. Philippe et al. Bortezomib as a new therapeutic approach for blastic plasmacytoid dendritic cell neoplasm. EHA abstracs 2017.

6. Pemmaraju et al. Ongoing phase 2 clinical trial of SL-401 in patients with blastic plasmacytoid dendritic cell neoplasm (BPDCN): stage 1 and stage 2 results. EHA abstracts 2017.

7. Gruson et al. L-asparaginase with methotrexate and dexamethasone is an effective treatment combination in blastic plasmacytoid dendritic cell neoplasm. British journal of Haematology 2013; 163. 